\title{
Effect of precursor solution dark incubation on gold nanorods morphology
}

\author{
Gaser N. Abdelrasoul a,b, Marco Scotto a, Roberto Cingolani ${ }^{a}$, Alberto Diaspro a,b, \\ Athanassia Athanassiou ${ }^{\mathrm{a}, \mathrm{c}}$, Francesca Pignatelli ${ }^{\mathrm{a}, *}$ \\ a Department of Nanophysics, Istituto Italiano di Tecnologia, via Morego 30, 16163 Genova, Italy \\ ${ }^{\mathrm{b}}$ University of Genova, via Balbi 5, 16126 Genova, Italy \\ c Center for Biomolecular Nanotechnologies@Unile, Istituto Italiano di Tecnologia (IIT), Via Barsanti, 73010 Arnesano (LE), Italy
}

\section{A R T I C L E I N F O}

\section{Article history:}

Received 5 March 2012

Received in revised form

4 September 2012

Accepted 6 September 2012

Communicated by K. Nakajima

Available online 14 September 2012

Keywords:

A1. Crystal morphology

A1. Nanostructures

A2. Growth from solutions

B1. Metals

Photochemical synthesis

\begin{abstract}
A B S T R A C T
Gold nanorods were synthesized in an aqueous solution of hexadecyltrimethylammonium bromide via a combination of chemical reduction and UV photoirradiation. Gold ligand complexes, present in the stock solution, are initially reduced, by ascorbic acid as mild reducing agent. The gold ions nucleation and colloid growth proceeds then by subsequent UV irradiation of the so-obtained precursor solution. We present a systematic study of the effect of incubation of the precursor solution on the dispersion state and aspect ratio of the produced nanorods. Incubation of the precursor solution allows the synthesis of higher aspect ratio nanorods with narrower size distribution compared to those obtained without incubation. We propose a mechanism for the gold nanorods formation including two stages, a nucleation and a diffusive growth. This allows us to explain the synthesis improvement as a consequence of the increase in the size of the gold ligand complexes aggregates, leading to a decrease of the nanorods growth rate.
\end{abstract}

(c) 2012 Elsevier B.V. All rights reserved.

\section{Introduction}

Gold nanorods (AuNRs) have received great attention in the past decade because of their unique optical [1,2] and electronic properties [3,4], which are influenced by their morphology. Generally, the properties of metal nanoparticles are determined by the type of motion that electrons in their conduction bands are allowed to execute. This motion depends on the space in which the electrons are confined and on the forces they encounter. In noble metals, their decrease in size below the electron mean free path (the distance the electron travels between scattering collisions with the lattice centers) gives rise to intense absorption in the visible-near-UV range [5]. This is the result of strong coupling of electromagnetic radiation of light at specific wavelengths to a surface plasmon (described as a coherent oscillation of the conduction electrons from one surface side of the particle to the other). The two dimensions in the nanometer size scale (width and length) in AuNRs give rise to two characteristic plasmon peaks, one located around $520 \mathrm{~nm}$, called the transverse surface plasmon peak (TSP), and the other in the near-infrared electromagnetic spectrum region at $600-800 \mathrm{~nm}$, called the longitudinal surface plasmon peak (LSP). These absorption peaks correspond to the oscillations of the transverse and longitudinal

\footnotetext{
* Corresponding author. Tel.: + 3901071781414 ; fax: + 390107170187

E-mail address: francesca.pignatelli@iit.it (F. Pignatelli).
}

plasmon modes along the short and long axes of the NRs, respectively. Different from the transverse plasmon absorption, longitudinal surface plasmon absorption is highly sensitive to the change in both the aspect ratio (length/width) of the NRs and the dielectric constant of the surrounding medium. This high sensitivity of the LSP peak makes AuNRs attractive candidates for biological applications [6] as biosensors [7-9], for cell imaging $[10,11]$, target drug delivery [12,13] and for nanomedicine $[14,15]$. AuNRs have interesting applications also in photonic devices [16,17], enhancement of Raman scattering spectroscopy [18], light fluorescence [19] and light scattering [20,21].

The large number of AuNRs applications has boosted scientists to develop different synthetic strategies, allowing the control of the dimensions, aspect ratio, and size distribution of AuNRs [22]. Several methods were adopted for synthesis of AuNRs including colloidal seed-mediated [23-26], hard template [27], electrochemical [28] and photochemical [29-32].

Between these methods the seed-mediated growth in aqueous media assisted by the surfactant hexadecyltrimethylammonium bromide (CTAB) and $\mathrm{Ag}^{+}$is undoubtedly the most popular, and has been deeply investigated [33]. Nevertheless, the mechanisms ruling the AuNRs anisotropic growth remain under debate. In the specific synthesis the growth solution is seeded by preformed gold seeds, directly after mixing its components. The formation of NRs starts 10 min after seed solution addition, but longer times (at least $6 \mathrm{~h}$ ) are needed to complete their growth. The anisotropic growth of AuNRs was found to depend on the size and 
concentration of the CTAB surfactant molecules [34] and it was first proposed to be governed by CTAB functioning as a soft micellar template [26]. Other researchers proposed preferential adsorption of bromide to different crystal faces during the AuNRs growth, inducing in this way nanoparticles elongation via a "zipping" mechanism [34]. Addition of silver nitrate to the seedmediated protocol was proposed in order to improve the size control and the yield of rod-shaped NRs [24]. The role of silver in assisting the anisotropic growth was explained through the underpotential deposition of metallic silver on the different crystal facets of gold [35]. A recent detailed study of the crystalline structure of AuNRs, prepared by silver assisted seed growth, proved that they are enclosed by eight identical high-index crystalline facets [36]. Consequent to these findings, the mechanisms commonly accepted for the anisotropic growth of AuNRs were revisited in favor of silver underpotential deposition on the different crystal facets, inducing lower growth rate on the higherenergy $\{250\}$-type surfaces, while the role of CTAB remains to be clarified.

Photochemical synthesis basically follows the procedure of seed-mediated synthesis, replacing seedings of gold nanoparticles with UV irradiation [31,32]. In this method the precursor solution, with composition similar to the growth solution of seed-mediated synthesis, is irradiated with UV light for 5-60 min, directly after mixing its components, in order to induce the nucleation of AuNRs. The photosynthesis approach is considered a promising evolution of the seed-mediated method, since it is less time consuming and avoids the use of seeds that can give rise to structure complications due to their crystallographic structure variety. However, the drawbacks of the photochemical synthesis are the polydispersity, heterogeneous size distribution, and small aspect ratio of the produced AuNRs. Many groups studied the factors affecting the growth, dispersion and aspect ratio of the AuNRs produced by photochemical method to understand the reaction mechanism of this protocol. Miranda et al. studied the effect of the light wavelength on the yield and aspect ratio of the AuNRs, and found that UV light at $300 \mathrm{~nm}$ produced longer NRs and with higher yield than at $254 \mathrm{~nm}$ [37]. They also studied the effect of UV light intensity and irradiation time on the aspect ratio and the dispersion of the NRs. The same group also studied the role of ascorbic acid in the growth of AuNRs, by evaluating the relation between concentration of ascorbic acid and gold salt precursor needed in order to produce AuNRs [38]. The presence of silver adsorbed on the surface of AuNRs produced by photochemical synthesis was also found to affect their aspect ratio and the anisotropic growth of specific crystal faces [39,40]. Finally, the effect of acetone addition to the reaction solution was studied by Nishioka et al., evaluating the function of ketones in the reduction of $\mathrm{Au}^{+}$ions with the assistance of UV light [41].

Here we propose for the first time dark incubation of the precursor solution before UV irradiation in order to equilibrate the aggregation state of the surfactant and to stress its role on the photochemical synthesis of AuNRs, assisted also by $\mathrm{Ag}^{+}$ions, in aqueous solution. According to literature, mixing the gold salt $\left(\mathrm{HAuCl}_{4}\right)$ in water with the CTAB surfactant induces the formation of complex ions $\mathrm{CTA}^{+} \mathrm{AuBr}_{4}^{-}[37,41]$. Further addition of ascorbic acid is known to reduce $\mathrm{Au}(\mathrm{III})$ to $\mathrm{Au}(\mathrm{I})$ [41]. Aggregates of $\mathrm{CTAB}$ and $\mathrm{AuBr}_{2}^{-}$laden $\mathrm{CTAB}$ are therefore expected to reorganize themselves after the addition of the ascorbic acid. Therefore, a stabilization time is required to equilibrate the solution. This observation triggered our investigation on the effect of incubation of the precursor solution in the dark before UV irradiation on the morphology of the NRs. The incubation in dark of the precursor solution provides the time necessary for its stabilization, with important consequences on the AuNRs aspect ratio. The precursor solution of AuNRs was incubated in dark without stirring for different time intervals, up to $4 \mathrm{~h}$, and then irradiated by UV light with wavelength of $254 \mathrm{~nm}$ for different times. The resulting NRs show significant aspect ratio enhancement, size distribution narrowing, and decreased yield, by increasing the time of incubation in dark. In particular, it was found that the length of the AuNRs increased while their diameter slightly decreased by incubation in dark. Light scattering and optical imaging measurements showed that the incubation of the precursor solution increases the dimensions of the CTAB surfactant aggregates, allowing us to associate their aggregation-state with the anisotropic NRs growth and their narrowed size dispersion after UV irradiation. Finally we propose a novel mechanism for rod formation that counts the role of the CTAB stabilizer in the increase of their aspect ratio and the improvement of their size distribution.

\section{Experimental section}

\subsection{Materials}

Hexadecyltrimethylammonium bromide CTAB (Fluka $\geq 99.0 \%$ ), hydrogen tetrachloroaurate trihydrate $\mathrm{HAuCl}_{4} \cdot 3 \mathrm{H}_{2} \mathrm{O}$ (Alfa Aeser 99.99\% (metal basis)), $\mathrm{Au} 49.5 \% \mathrm{~min}$ ), and silver nitrate $\mathrm{AgNO}_{3}$ (Sigma Aldrich 99.9999\%) were used without further purification. Ascorbic acid (99\%), acetone and cyclohexane were purchased from Sigma-Aldrich and used as received. All solutions were prepared with Milli-Q double distilled water (resistivity $>18.2 \mathrm{M} \Omega \mathrm{cm}$ ).

\subsection{Synthesis}

To $3.320 \mathrm{~mL}$ of reaction solution, containing $24 \times 10^{-5} \mathrm{~mol}$ of CTAB, $0.6 \times 10^{-5} \mathrm{~mol}$ of $\mathrm{HAuCl}_{4} \cdot 3 \mathrm{H}_{2} \mathrm{O}$ and $0.07 \times 10^{-5} \mathrm{~mol}$ of $\mathrm{AgNO}_{3}$, were added $0.065 \mathrm{~mL}$ of acetone and $0.045 \mathrm{~mL}$ of cyclohexane. The color of this solution was deep orange, and after addition of $0.2 \mathrm{~mL}$ of ascorbic acid $(40 \mathrm{mM})$ it became colorless within $1 \mathrm{~min}$.

$3 \mathrm{~mL}$ of precursor solution was put into a quartz cuvette of optical path length $1 \mathrm{~cm}$, and irradiated with UV light from an ultrahigh-pressure mercury lamp (Oriel $6035 \mathrm{Hg}(\mathrm{Ar})$ ) with calibrated light wavelength of $254 \mathrm{~nm}$, for $1,5,10$, and $30 \mathrm{~min}$. The average irradiation intensity was kept at $2.5 \mathrm{~mW} / \mathrm{cm}^{2}$ during all the experimental work. In order to study the effect of incubation time in the dark on the resulting AuNRs, the remaining precursor solution was kept in the dark for different periods before UV irradiation. After each incubation period of study (1, 2, 3 and $4 \mathrm{~h}$ ) $3 \mathrm{~mL}$ of the precursor solution was taken, put in a quartz cuvette and irradiated.

The synthesis was performed at room temperature, about $25^{\circ} \mathrm{C}$. The intensity of the UV light was measured before and after irradiation of the precursor solution, by a power meter Thorlab PM150, to get the average value of irradiation intensity. The quartz cuvette containing the reaction solution and the UV lamp were covered with a black box during the irradiation to prevent any possible interaction with other light sources in the lab. After irradiation the reaction solution was centrifuged at $4000 \mathrm{rpm}$ for $10 \mathrm{~min}$ to separate the big gold clusters. The resulting supernatant was collected and centrifuged at 14,000 rpm each for $20 \mathrm{~min}$. The precipitate was collected and washed twice to discard the excess of CTAB and the unreacted materials from the solution. The supernatant was exchanged with double distilled water and the final precipitate was dispersed in $1 \mathrm{~mL}$ of distilled water and used for characterization. 


\subsection{Characterization}

The hydrodynamic diameters of the surfactant aggregates in the precursor solution were measured by a light scattering Malvern Zeta Sizer NanoZS equipped with $633 \mathrm{~nm}$ He-Ne laser. A time lapse imaging acquisition was done using the transmission channel of a Laser Scanning Confocal Microscope (LSCM, Nikon A1). After the separation and washing processes, $20 \mu \mathrm{L}$ of NRs solution dispersed in water were deposited on carbon coated copper grid and dried under vacuum for transmission electron microscopy (TEM) investigation. The transmission electron micrographs were taken by a JEOL JEM1011 at $100 \mathrm{kV}$. For optical characterization, $100 \mu \mathrm{L}$ of each AuNRs solution was diluted to $400 \mu \mathrm{L}$ with Milli-Q water in quartz cuvette with optical path length $2 \mathrm{~mm}$ for absorption spectral analysis. The absorption spectra of all AuNRs solutions were collected using a UV-vis-NIR spectrophotometer Varian Cary 6000i. Gold and silver ion concentrations were determined by a Thermo Fisher Scientific Inductively coupled plasma (ICP, ICAP 6300 DUO) atomic emission spectrometer. For ICP measurements, $50 \mu \mathrm{L}$ of AuNRs solution was digested by the addition of $2.5 \mathrm{~mL}$ of aqua regia in $25 \mathrm{~mL}$ plastic measuring flask.

\section{Results and discussion}

Following the procedures outlined in the experimental section we prepared AuNRs by UV irradiation of their precursor solution, after different dark incubation times of the latter prior to the UV treatment. No color change of the precursor solution was recorded during dark incubation, indicating that nucleation of AuNRs does not start during the incubation of the precursor solution in dark. This confirms that ascorbic acid is a mild reducing agent, not able to start the NRs nucleation by reducing $\mathrm{Au}^{+}$ions to $\mathrm{Au}^{0}$ atoms, in absence of UV irradiation [42]. The effect of incubation of the precursor solution in the dark before UV irradiation on the surfactant-based aggregates size was preliminary investigated by dynamic light scattering and optical imaging. The dynamic light scattering measurements showed that the diameter of the aggregates in the precursor solution increases with increasing incubation time from about 100 to $500 \mathrm{~nm}$. At the same time the dispersion state of the precursor solution is found to increase from $<0.5$ to about 0.9 after $4 \mathrm{~h}$ of incubation. In agreement with light scattering results, optical imaging measurements highlighted presence of big aggregates in the precursor solution increasing in size and in number with the progress of the dark incubation, as demonstrated in the Supporting data.

The precursor solution incubated for different time intervals was then irradiated with UV light for different times, obtaining AuNRs. Characteristic TEM images of the final outcome of the synthesis after $30 \mathrm{~min}$ are shown in Fig. 1, where it is clear that the amount of the produced spherical nanoparticles is reduced in favor of the uniform AuNRs, as the incubation time increases.
Further TEM analysis gives detailed information on the size distribution and the dispersion of the AuNRs. The effect of incubation in dark on AuNRs morphology has been estimated by the respective histograms, obtained from the statistics over the dimensions of 100 NRs.

As shown in Fig. 2, the frequency of the NRs with short lengths decreases by increasing the incubation time in dark. In detail, the shortest NRs with length between 20 and $25 \mathrm{~nm}$, available in the non-incubated sample disappear completely in the samples obtained after a $2 \mathrm{~h}$ incubation or more. The frequency of the AuNRs with length in the range between 30 and $35 \mathrm{~nm}$ increases from 0.13 in the case of non-incubated sample to 0.25 with $1 \mathrm{~h}$ of incubation in the dark. However, their frequency decreases for longer incubation times in the dark. This decrease seems to be for the benefit of longer NRs. AuNRs with length in the range of $35-40 \mathrm{~nm}$ are more frequent in the solutions whose precursors were not incubated or incubated for 1 and $2 \mathrm{~h}$. The frequency of AuNRs with length between 40 and $45 \mathrm{~nm}$ increases from 0.15 for AuNRs solutions whose precursors were not incubated, to become predominant with frequency more than 0.35 after $4 \mathrm{~h}$ of incubation. The longest NRs with length between 60 and $70 \mathrm{~nm}$ started to be observed with small frequency only after $4 \mathrm{~h}$ of incubation in dark before UV irradiation. Also, it is noted that, the dispersion of AuNRs lengths was immediately narrowed upon precursor incubation even for one hour compared to the non-incubated solutions. The standard deviation of the lengths decreases from $3 \mathrm{~nm}$ in case of non-incubated sample to $1 \mathrm{~nm}$ in case of $4 \mathrm{~h}$ incubation, indicating the crucial role of the incubation of the precursor solution in dark before UV irradiation on the dispersion state of the resulted NRs. Fig. $2 \mathrm{f}$ shows the relationship between the incubation times of the AuNRs precursor solutions in the dark before UV irradiation and the mean length of the resulting NRs. The latter linearly increases with the incubation time of the precursor solution before UV irradiation, with a rate of about $2 \mathrm{~nm} / \mathrm{h}$. As overall effect the mean AuNRs length increases from $\sim 36$ to $44 \mathrm{~nm}$ after a $4 \mathrm{~h}$ incubation.

Fig. 3 presents the histograms of AuNRs diameter distribution obtained after $30 \mathrm{~min}$ UV irradiation of the precursor solution, previously incubated in the dark for different times. Generally, a small decrease in the diameters of the AuNRs was observed with increasing incubation time. In detail, NRs with diameter between 18 and $20 \mathrm{~nm}$ disappear after $1 \mathrm{~h}$ of incubation. The frequency of AuNRs with a bit smaller diameter in the range of $16-18 \mathrm{~nm}$ decreases gradually with increasing incubation time and becomes zero after $4 \mathrm{~h}$ incubation. A similar trend was observed for the NRs having diameter between 14 and $16 \mathrm{~nm}$. Their frequency varies from 0.15 for the non-incubated samples to 0.01 after $4 \mathrm{~h}$ of incubation. On the other hand, the number of NRs with smaller diameters increases with increasing incubation time in the dark of their precursor solutions before UV irradiation. For example, the frequency of the AuNRs with diameter between 8 and $10 \mathrm{~nm}$ increases from 0.14 for the non-incubated samples to a maximum
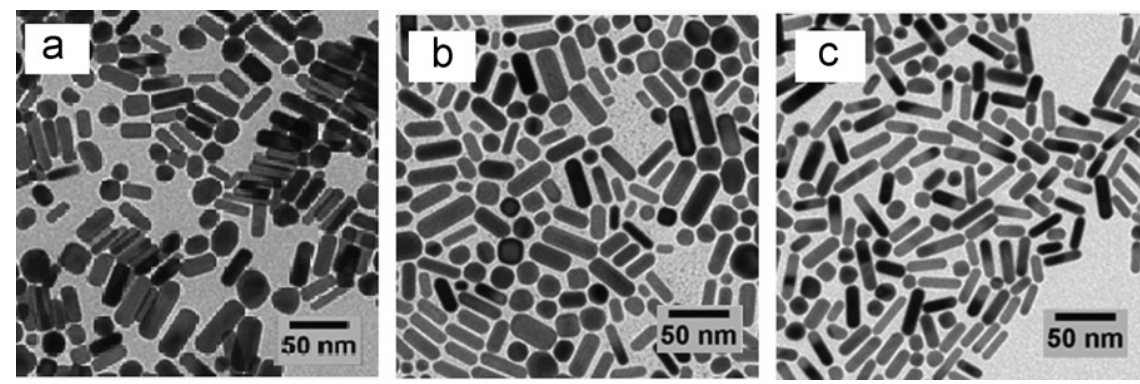

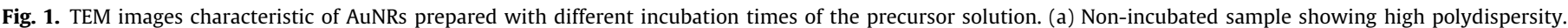
Samples obtained with dark incubation of precursor solutions, (b) $2 \mathrm{~h}$ and (c) $4 \mathrm{~h}$, showing NRs with improved dispersion state and aspect ratio. 

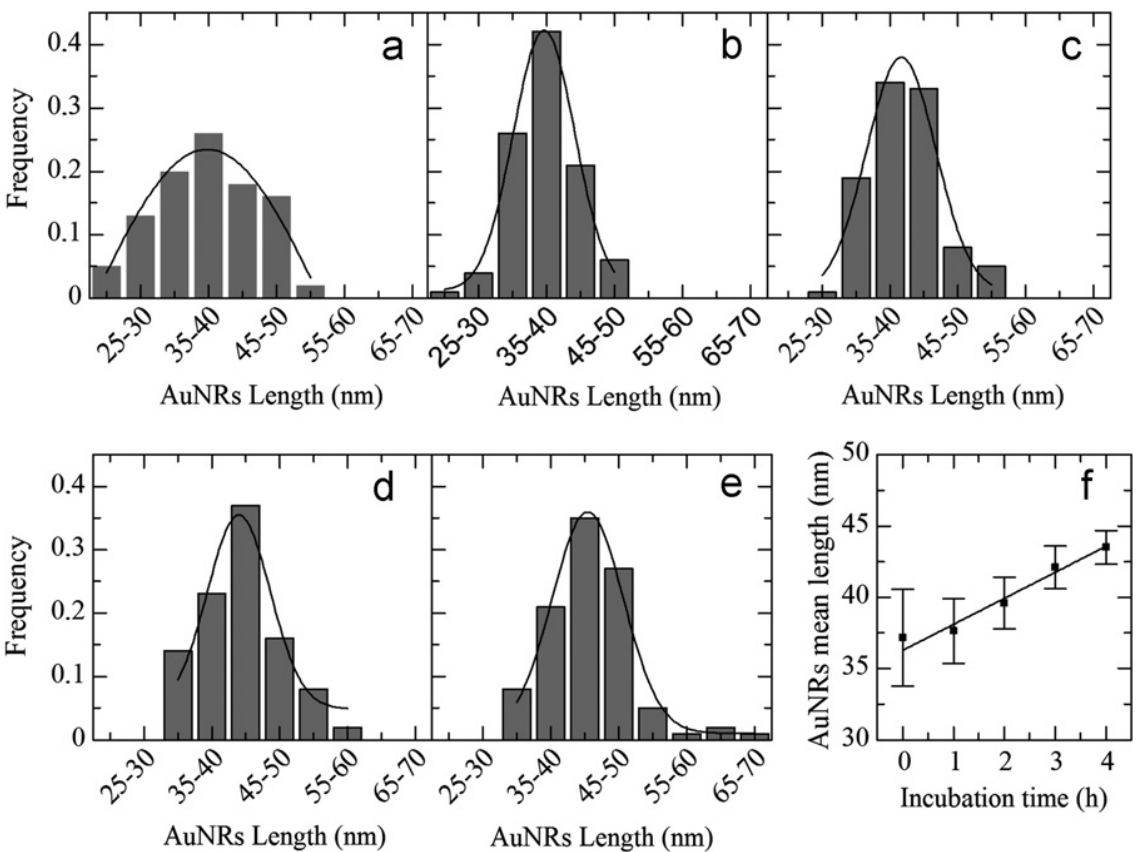

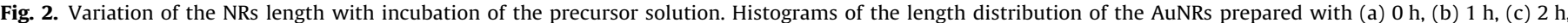

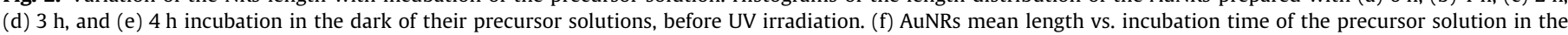
dark.

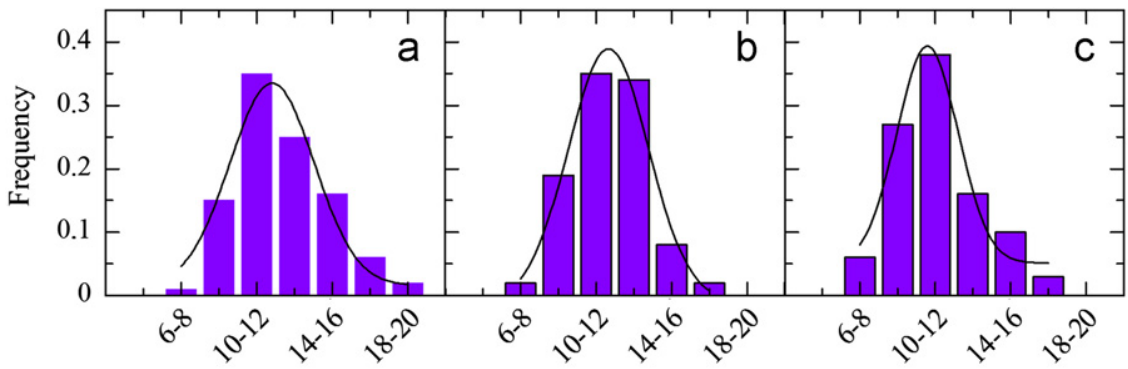

AuNRs Diameter (nm)

AuNRs Diameter (nm)

AuNRs Diameter (nm)
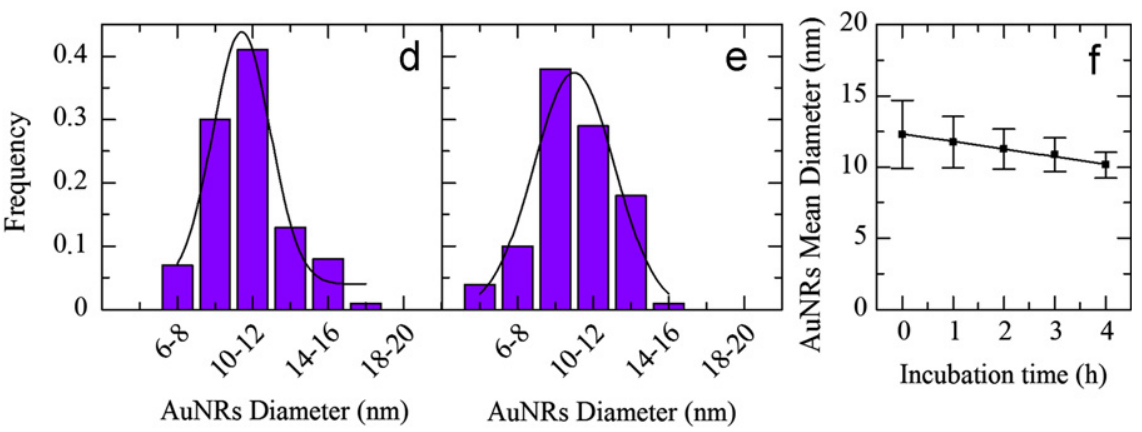

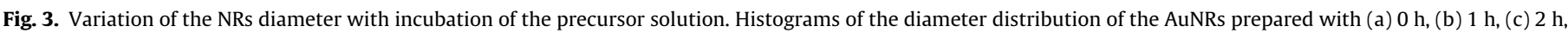

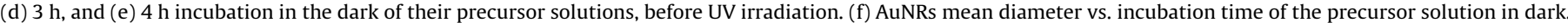

of 0.38 after $4 \mathrm{~h}$ of incubation. The narrowest NRs with diameter in the range between 4 and $6 \mathrm{~nm}$ are observed only after $4 \mathrm{~h}$ of precursor solution incubation in dark. The AuNRs diameter histograms also show that the diameters dispersion tends to narrow with increasing incubation time of the precursor solution in dark, before UV irradiation. In AuNRs samples obtained by irradiating a non-incubated precursor solution, the value of the standard deviation of AuNRs diameters was $2 \mathrm{~nm}$. Whereas the sample obtained irradiating the precursor solution, after $4 \mathrm{~h}$ incubation, has a standard deviation of the diameters around
$1 \mathrm{~nm}$, indicating the enhancement of monodispersion of the resulted AuNRs.

As previously done for the mean length, Fig. 3f shows the dependence of the AuNRs mean diameter on the incubation time in the dark of their precursor solution. The AuNRs diameter linearly decreases with increasing incubation time. The slope of the linear fit of the decrease of AuNRs diameter with incubation time is $-0.5 \mathrm{~nm} / \mathrm{h}$, opposite in sign and smaller than the respective one for the increase of the AuNRs length. The simultaneous increase of the length and decrease of the diameter of the formed AuNRs, leads 

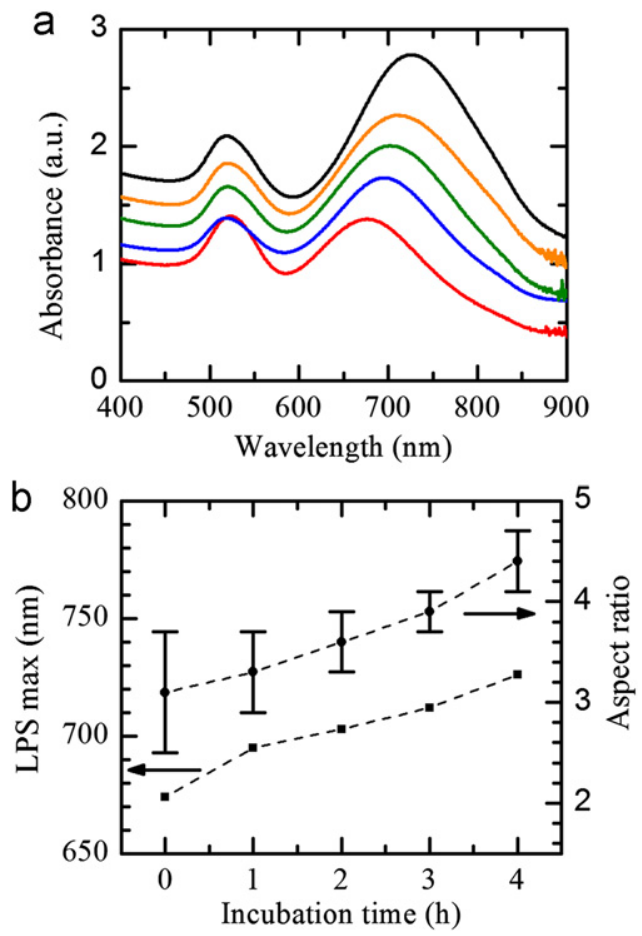

Fig. 4. Effect of precursor solution incubation on the aspect ratio of the produced NRs. (a) Absorption spectra of the formed AuNRs solutions obtained by different dark incubation times of the precursor solution before irradiation and (b) maximum absorption of the AuNRs LSP and aspect ratios, estimated from TEM analysis, vs. incubation time.

to the increase of their aspect ratio with increasing incubation time of the solutions precursors in the dark. The aspect ratios calculated from the TEM images is reported in Fig. 4b.

The effect of incubation of the precursor solution in dark on the AuNRs morphology is further corroborated by the UV-vis spectra of the resulting AuNRs solutions after 30 min of irradiation of their precursor solution. Fig. 4a shows the UV-vis absorption spectra of AuNRs solutions whereas Fig. 4b demonstrates the behavior of LSP peak position and NRs aspect ratio with precursor solution incubation time in dark. The shape of the absorption peaks indicates that the AuNRs solutions have narrow size dispersion. As mentioned in the introduction, the absorption spectrum of AuNRs is characterized by two plasmonic absorption peaks, the TSP centered around $520 \mathrm{~nm}$, less sensitive to the NRs aspect ratio and the dielectric constant of the surrounding medium, and the LSP at higher wavelengths, highly sensitive both to the dielectric constant of the medium and the aspect ratio of the NRs. As the AuNRs aspect ratio increases the LSP is expected to redshift to longer wavelengths. In our case, considering the same dispersing medium, we observe redshift of the LSP absorption peak wavelength with increasing the incubation time in the dark of the AuNRs precursor solutions. The optical absorption points out an increase of the resulting AuNRs aspect ratio in agreement with TEM measurements. Furthermore, in Fig. 4a it can also be noted that the TSP and LSP peaks, in the case of nonincubated samples, have almost the same absorption intensities, which indicates the presence of many spherical gold nanoparticles even after purification and washing. For the other samples, the LSP intensity is higher than the TSP one, with the difference to be maximized for the samples obtained after $4 \mathrm{~h}$ of dark incubation, indicating the favorable formation of NRs over the spherical nanoparticles for longer incubation times.

In order to clarify the mechanism leading to NRs synthesis improvement upon dark incubation of the precursor solution, we studied the progress of the NRs formation after 1,5 , and 10 min of irradiation, in the two extreme conditions of nonincubation and of $4 \mathrm{~h}$ incubation. This allowed us to resolve the modifications during the UV-induced growth of the NRs after incubation of the precursor solution. The absorbance spectra for the non-incubated and incubated solutions after 1,5 and 10 min are reported respectively in Fig. 5(a-c).

The evolution of the synthesis upon UV irradiation, as highlighted by the absorbance spectra, points out similar trends but also significant differences for the non-incubated and incubated samples. In both cases the absorption intensity increases with increasing irradiation time, pointing out the increasing formation of nanocrystals in the investigated time range. On the top, with the prolongation of the irradiation time the intensity of the LSP peak raises more intensively than the one of the TSP peak, suggesting the presence of increasing number of anisotropic nanoparticles with respect to the spherical ones. At the same
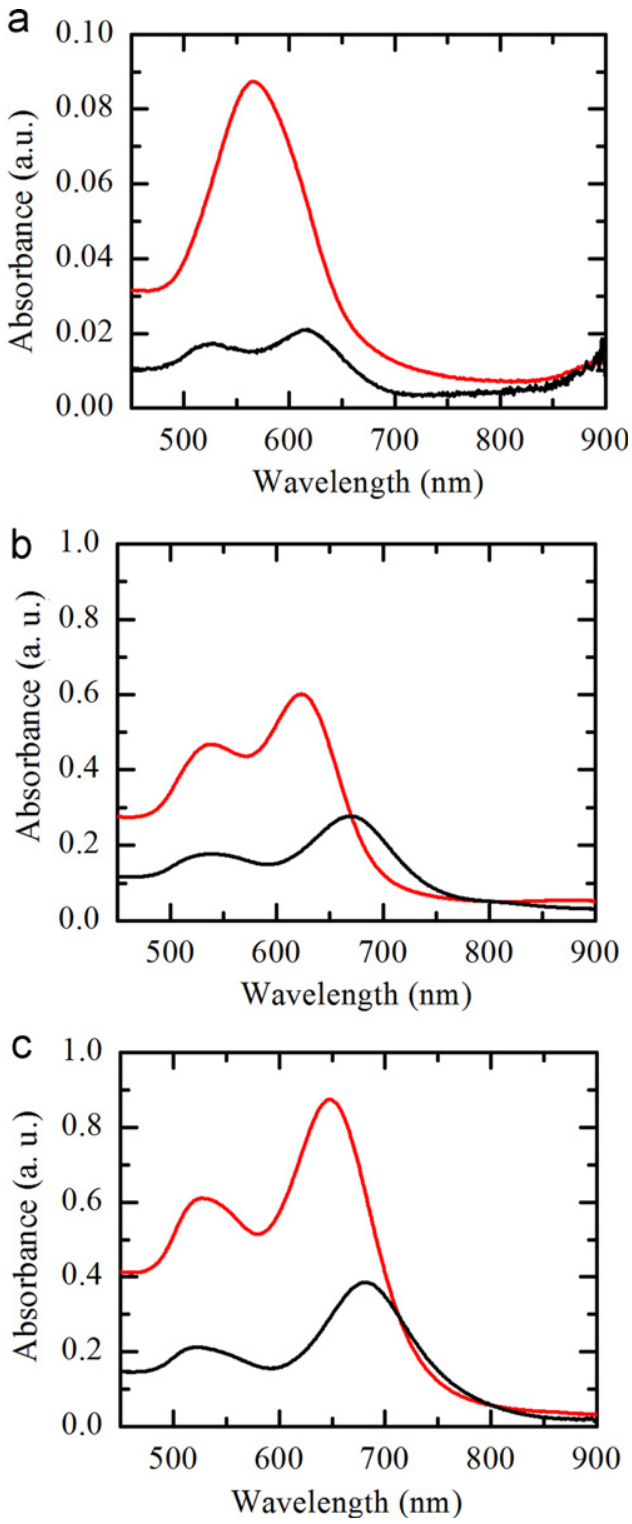

Fig. 5. Absorption spectra of the formed AuNRs solutions after different times of irradiation: (a) $1 \mathrm{~min}$, (b) $5 \mathrm{~min}$ and (c) $10 \mathrm{~min}$. Red and black lines correspond respectively to non-incubated precursor solutions and incubated for $4 \mathrm{~h}$ (For interpretation of the references to color in this figure legend, the reader is referred to the web version of this article). 
time the wavelengths of the LSP peaks redshift with the progress of the irradiation. Therefore, on the top of higher NRs yield, the aspect ratio of the formed rods increases extending the irradiation time. For all three irradiation times, the non-incubated samples present higher absorbance intensities, indicating higher synthesis yields for the non-incubated samples. Furthermore, the relative increase of the LSP peak intensity with respect to the TSP one is higher for the non-incubated samples compared to the incubated ones, pointing out a higher AuNRs yield in case of non-incubation of the precursor solution. Overall the incubation of the precursor solution reduces the AuNRs production yield while favors their anisotropic growth.

The effect of the incubation in dark on the NRs' production yield was evaluated also from the ICP measurements. The concentration of $\mathrm{Au}^{+}$ions in the resulting AuNRs solution after $30 \mathrm{~min}$ irradiation decreased from $1.006 \mathrm{ppm}$ for the nonincubated sample to $0.5724 \mathrm{ppm}$ for the sample incubated for $4 \mathrm{~h}$. A similar behavior was observed for the concentration of the $\mathrm{Ag}+$ ions decreasing from $0.0395 \mathrm{ppm}$ in the non-incubated sample to $0.013 \mathrm{ppm}$ for the sample incubated $4 \mathrm{~h}$. The decrease of the amount of gold and silver ions in the final NRs solution, induced by incubation of the precursor solution before UV irradiation, suggests a decrease of the growth yield of the NRs, in agreement with results obtained by the optical characterization.

Sugimoto pointed out that a key point in order to achieve narrow size distribution of nanoparticles is a good control, during the synthesis, of nucleation and growth [43]. In particular, the ideal condition would be to separate the two steps, having a fast nucleation followed by a slow growth. The fast nucleation would ensure nuclei of same sizes whereas the slow growth would ensure a similar growth rate for all produced particles. Differently from the seed-mediated synthesis, in the photochemical strategy for the synthesis of AuNRs, the nucleation of the Au seeds and their growth occur by UV irradiation in just one step. This is the main disadvantage of photochemical synthesis with respect to the seed-mediated protocol, leading to polydispersion and in general lower aspect ratios of the obtained particles. At high concentrations, as in our experimental conditions, CTAB in water is known to give rise to aggregates. Although high $\mathrm{CTAB}$ concentrations were shown to be critical for nanorods production [24], recent works $[36,40]$ showed that the anisotropic growth is highly affected by the so-called underpotential deposition (UDP) of metallic silver on the different crystal facets decreasing the $\mathrm{Au}$ growth rate on the lateral faces. Herein we focus on the role of CTAB in helping the anisotropic growth of the NRs after the precursors' incubation in the dark.

It is well known that CTAB functions as stabilizer of the produced NRs in the aqueous solution. Furthermore CTAB contributes to the photochemical synthesis by making complexes with gold ions. In particular, the $\mathrm{AuCl}_{4}^{-}$ions in the precursor solution are expected to first displace $\mathrm{Br}^{-}$ions and then bind tightly to $\mathrm{CTA}^{+}$forming $\mathrm{CTA}^{+} \mathrm{AuBr}_{4}^{-}$complexes [37]. Further addition of ascorbic acid induces reduction of the $\mathrm{CTA}^{+} \mathrm{AuBr}_{4}^{-}$ complexes to $\mathrm{CTA}^{+} \mathrm{AuBr}_{2}^{-}$complexes. The high $\left[\mathrm{CTA}^{+}\right]:\left[\mathrm{AuCl}_{4}^{-}\right]$ molar ratio in the reaction solution needed for our synthesis (40:1) suggests that all Au precursors gets tightly bound to $\mathrm{CTA}^{+}$, forming $\mathrm{CTA}^{+} \mathrm{AuBr}_{2}^{-}$complexes. Therefore, the CTAB beyond its stabilizing character, functions as precursor for the $\mathrm{CTA}^{+} \mathrm{AuBr}_{2}^{-}$ complexes. We propose that a further role of the CTAB is to ensure good gold ions reservoirs through the big aggregates of CTAB and $\mathrm{CTA}^{+} \mathrm{AuBr}_{2}^{-}$, formed in the precursor solutions. The reservoirs keep the concentration of free gold ions dispersed in the solution low during the growth and at the same time lower the speed of their movement due to their complexation in big aggregates. Therefore, the aggregation state of the CTAB and
$\mathrm{CTA}^{+} \mathrm{AuBr}_{2}^{-}$is expected to influence the nucleation, growth and dispersion of the later formed AuNRs.

UV irradiation of the dark incubated precursor solutions generates ketyl radicals via excitation of acetone and hydrogen atom abstraction from CTAB [41]. These radicals are strong reductants and react with each other or initiate reduction of $\mathrm{AuBr}_{2}^{-}$ions to $\mathrm{Au}(0)$ [41]. A similar reaction of ketyl radicals with silver ions would be also possible but with slower rate, because of lower electrochemical potential and lower concentration of silver ions. Next, the $\mathrm{Au}(0)$ accumulate up to a critical concentration at which nuclei will start to form, giving rise to the primary colloidal particles (nucleation), which are then stabilized by a CTAB bilayer, according to previous results [34,44]. Once a small number of gold colloids are formed, a second reduction process can occur by their collision with the gold ions reservoirs. This reduction occurs in two steps. First ketyl radicals transfer electrons to the colloidal gold particles [45]. Then stored electrons induce $\mathrm{AuBr}_{2}^{-}$and $\mathrm{Ag}^{-}$ ions reduction at the surface of the colloidal particles. This reaction, competing with the first one during the growth of the colloids, is diffusion controlled. In particular for silver ions the diffusion controlled reaction was estimated to be predominant, being three time faster than the other [46].

According to kinetic theory, the number and size of the particles in solution determine their probability of collision in the course of their Brownian motion. Therefore the diffusion controlled growth of each formed gold particle depends on its dimensions and on population and dimensions of gold ions reservoirs in the reaction solution. In particular, the bigger the reagents the slower the diffusion controlled growth rate. In the case of fixed concentrations of the reagents of the synthesis the number of gold ions reservoirs and the speed of their Brownian motion in the precursor solution are both ruled by the aggregation state of $\mathrm{CTAB}$ and $\mathrm{CTA}^{+} \mathrm{AuBr}_{2}^{-}$. As proved above in our experiments the size of these aggregates increases with increasing incubation time.

Moreover, slow collision frequencies are expected to support anisotropic growth by preferential reduction of the gold precursor at the tips of the NRs due to potential drop near the tips [47]. Electrons stored at the surface of the AuNRs are expected to induce a potential distribution outside the rod decaying more rapidly near the tips. This electric field distribution facilitates aggregate collisions at the tips rather than at the sides. The anisotropic growth becomes more prominent, reducing the speed of approaching aggregates in agreement with previous experimental results [37]. Along the progress of the AuNRs synthesis the formed particles growth, therefore increase in size and therefore their motion slow up, whereas the dimensions of the reservoirs keep constant. Thus increasing the irradiation time the reduction in the collision rate own favors the anisotropic growth, provided the concentration of gold ions in the solution is sufficient to avoid Ostwald ripening. This explains the observed enhancement of the aspect ratio with increasing irradiation time. On the other side incubation of the precursor solution results in the increase of the $\mathrm{CTAB}$ and $\mathrm{CTA}^{+} \mathrm{AuBr}_{2}^{-}$complexes aggregate dimensions, inducing through the decrease of the speed of the gold ions reservoirs collisions improvement in the anisotropic growth and in the dispersion of the NRs.

\section{Conclusions}

The influence on nanorod morphology of dark incubation of the precursor solution has been investigated for the first time. The precursor solution for AuNRs photochemical synthesis consisted of $\mathrm{HAuCl}_{4} \cdot 3 \mathrm{H}_{2} \mathrm{O}$ as the gold salt precursor, CTAB as surfactant, $\mathrm{AgNO}_{3}$ as uniaxial growth directing agent, cyclohexane as micelle 
stabilizer, acetone as free radical initiator and ascorbic acid as mild reducing agent. After the first reaction step, involving the reduction of $\mathrm{Au}(\mathrm{III})$ complexes to $\mathrm{Au}(\mathrm{I})$ complexes under the effect of the addition of the ascorbic acid to the precursor solution, the reaction solution was incubated in the dark for different intervals. The effect of incubation in dark before UV irradiation on the aspect ratio and the dispersion state of the AuNRs was studied by complementary TEM and UV-vis-NIR absorption measurements. It was found that, as the incubation time for the precursor solution in dark increases the dimensions of CTAB and $\mathrm{CTA}^{+} \mathrm{AuBr}_{2}^{-}$aggregates, the aspect ratio of the resulting NRs also increases, due to a simultaneous increase of the length and a small decrease of the width of the NRs. Also, the size distribution of the AuNRs was significantly narrowed by incubation of the AuNRs precursor solutions in the dark before UV irradiation.

We propose a growth mechanism to account for our results that considers $\mathrm{CTAB}$ and $\mathrm{CTA}^{+} \mathrm{AuBr}_{2}^{-}$aggregates as precursor reservoirs. In particular, larger aggregates induce slower diffusive growth rates that besides favoring anisotropic growth also reduce the size dispersion of the formed rods.

\section{Acknowledgment}

We thank Dr. Liberato Manna, Nanochemistry group, at the Italian Institute of Technology for discussions.

\section{Appendix A. Supporting information}

Supplementary data associated with this article can be found in the online version at http://dx.doi.org/10.1016/j.jcrysgro.2012. 09.009 .

\section{References}

[1] S. Link, M.B. Mohamed, M.A. El-Sayed, Journal of Physical Chemistry B 103 (1999) 3073.

[2] X. Huang, S. Neretina, M.A. El-Sayed, Advanced Materials 21 (2009) 4880

[3] M. Chirea, A. Cruz, C.M. Pereira, A.F. Silva, Journal of Physical Chemistry C 113 (2009) 13077

[4] M. Chirea, J. Borges, C.M. Pereira, A.F. Silva, Journal of Physical Chemistry C 114 (2010) 9478

[5] M.A. El-Sayed, Accounts of Chemical Research 34 (2001) 257.

[6] J. Stone, S. Jackson, D. Wright, WIREs Nanomedicine and Nanobiotechnology 3 (2011) 100

[7] C. Wang, J. Irudayaraj, Small 4 (2008) 2204

[8] K.S. Anant, S. Dulal, W. Shuguang, G. Jelani, N. Adria, C. Perry, M.N. Khaleah V. Birsen, R.K. Jhansi, C. Paresh, Ray, American Chemical Society Nano 3 (2009) 1906.

[9] N. Omura, I. Uechi, S. Yamada, Analytical Sciences 25 (2009) 255.
[10] L. Tong, Q. Wei, A. Wei, J.X. Cheng, Photochemistry and Photobiology 85 (2009) 21

[11] T. Niidome, Y. Akiyama, K. Shimoda, T. Kawano, T. Mori, Y. Katayama, Y. Niidome, Small 4 (2008) 1001.

[12] Q. Wei, J. Ji, J. Shen, Macromolecular Rapid Communications 29 (2008) 645.

[13] T.R. Kuo, V.A. Hovhannisyan, Y.C. Chao, S.L. Chao, S.J. Chiang, S.J. Lin, C.Y. Dong, C.C. Chen, Journal of the American Chemical Society 132 (2010) 14163.

[14] W.S. Kuo, C.N. Chang, Y.T. Chang, M.H. Yang, Y.H. Chien, S.J. Chen, C.S. Yeh, Angewandte Chemie-International Edition 49 (2010) 2711.

[15] C. Nripen, R. Shukla, K.V. Katti, R. Kannan, Nano Letters 9 (2009) 1798

[16] A. Mohammadi, V. Sandoghdar, M. Agio, New Journal of Physics 10 (2008) 105015.

[17] B. Nikoobakht, US patent, Appl. Publi (2010).

[18] Y. Wang, S. Guo, H. Chen, E. Wang, Journal of Colloid and Interface Science 318 (2008) 82

[19] S. Eustis, M.A. El-Sayed, Journal of Physical Chemistry B 109 (2005) 16350.

[20] L. Qiu, T.A. Larson, E. Vitkin, L. Guo, E.B. Hanlon, I. Itzkan, K.V. Sokolov, L.T. Perelman, Biomedical Optics Express 1 (2010) 135.

[21] C.J. Orendorff, S.C. Baxter, E.C. Goldsmith, C.J. Murphy, Nanotechnology 16 (2005) 2601

[22] J. Pérez-Juste, I. Pastoriza-Santos, L.M. Liz-Marzán, P. Mulvaney, Coordination Chemistry Reviews 249 (2005) 1870.

[23] B. Nikoobakht, M.A. El-Sayed, Chemistry of Materials 15 (2003) 1957.

[24] T.K. Sau, C.J. Murphy, Langmuir 20 (2004) 6414.

[25] Z.L. Wang, M.B. Mohamed, S. Link, M.A. El-Sayed, Surface Science 440 (1999) L809.

[26] N.R. Jana, L. Gearheart, C.J. Murphy, Journal of Physical Chemistry B 105 (2001) 4065

[27] Z. Li, C. Kubel, V.I. Parvulescu, R. Richards, American Chemical Society Nano 2 (2008) 1205

[28] Y.Y. Yu, S.S. Chang, C.L. Lee, C.R.C. Wang, Journal of Physical Chemistry B 101 (1997) 6661.

[29] K. Torigeo, K. Esumi, Langmuir 8 (1992) 59.

[30] K. Esumi, K. Matsuhisa, K. Torigoe, Langmuir 11 (1995) 3285

[31] F. Kim, J.H. Song, P. Yang, Journal of the American Chemical Society 124 (2002) 14316.

[32] Y. Niidome, K. Nishioka, H. Kawasaki, S. Yamada, Chemical Communications (2003) 2376

[33] C.J. Murphy, L.B. Thompson, D.J. Chernak, J.A. Yang, S.T. Sivapalan, S.P. Boulos, J. Huang, A.M. Alkilany, P.N. Sisco, Current Opinion in Colloid and Interface Science 16 (2011) 128 .

[34] J. Gao, M.B. Christopher, C.J. Murphy, Langmuir 19 (2003) 9065.

[35] M.Z. Liu, P.J. Guyot-Sionnest, Journal of Physical Chemistry B 109 (2005) 22192.

[36] E. Carbó-Argibay, B. Rodríguez-González, S. Gómez-Graña, A. GuerreroMartínez, I. Pastoriza-Santos, J. Pérez-Juste, L. Liz-Marzán, Angewandte Chemie 122 (2010) 9587.

[37] O.R. Miranda, T.S. Ahmadi, Journal of Physical Chemistry B 109 (2005) 15724.

[38] O.R. Miranda, R.N. Dollahon, T.S. Ahmadi, Crystal Growth and Design 6 (2006) 2747.

[39] Y. Niidome, Y. Nakamura, K. Honda, Y. Akiyama, K. Nishioka, H. Kawasaki, N. Nakashima, Chemical Communications (2009) 1754.

[40] T. Placido, R. Comparelli, F. Giannici, P.D. Cozzoli, G. Capitani, M. Striccoli, A. Agostiano, L. Curri, Chemistry of Materials 21 (2009) 4192.

[41] K. Nishioka, Y. Niidome, S. Yamada, Langmuir 23 (2007) 10353.

[42] A. Gole, C.J. Murphy, Chemistry of Materials 16 (2004) 3633.

[43] T. Sugimoto, Advances in Colloid and Interface Science 28 (1987) 65

[44] B. Nikoobakht, M.A. El-Sayed, Langmuir 17 (2001) 6368.

[45] A. Henglein, J. Lilie, J. Am. Soc. 103 (1981) 1059.

[46] A. Henglein, Chemistry of Materials 10 (1998) 444.

[47] J. Pérez-Juste, L.M. Liz-Marzán, S. Carnie, D.Y.C. Chan, P. Mulvaney, Advanced Functional Materials 14 (2004) 571. 